\section{P16 NON-INVASIVE EVALUATION OF FIBROSIS IN PAEDIATRIC CHRONIC LIVER DISEASE}

doi:10.1136/gutjnl-2011-300857a.16

${ }^{1}$ E Fitzpatrick, ${ }^{1} \mathrm{M}-\mathrm{S}$ Basso, ${ }^{2} \mathrm{~A}$ Quaglia, ${ }^{2} \mathrm{R}$ R Mitry, ${ }^{1} \mathrm{~A}$ Dhawan. ${ }^{1}$ Paediatric Liver, Gl and Nutrition Centre, King's College Hospital; ${ }^{2}$ Institute of Liver Studies, King's College Hospital

Introduction Outcome of liver disease in children is mainly determined by severity and progression of liver fibrosis. Liver biopsy is the accepted standard for evaluating fibrosis but is limited by the need for sedation in children, sampling error and risks including bleeding. Aim The aim of this study was to compare tools for non-invasive assessment of liver fibrosis in a paediatric cohort.

Method Children were recruited at the time of liver biopsy and underwent transient elastography (TE) and blood collection. Liver biopsies were scored by a hepato-histopathologist from F0 (no fibrosis) to F4 (cirrhosis). Serum samples underwent analysis for the Enhanced Liver Fibrosis (ELF) test; comprising hyaluronic acid, P3NP and TIMP1 (iQur, UK). CK18-M30 levels (caspase cleavage fragments) were measured using ELISA. Biomarkers were compared to biopsy score. Results During the study period 101 children (62 boys) were enrolled. Median age: 13.6 (range 6-18) years. Diagnosis was autoimmune liver disease (AILD) in 27; non-alcoholic fatty liver disease (NAFLD) in 37; 13 children were post-transplant; 8 children had Hepatitis B/C; 5 had Wilson disease and the remainder miscellanous. TE was a good discriminator of fibrosis $\geq$ F2 $(p<0.001), \geq F 3(p<0.001)$ and F4 $(p=0.003)$ with areas under the ROC curve of $0.78,0.8$ and 0.96 respectively. Data derived cut-offs for $\geq \mathrm{F} 1$ were $6.1 \mathrm{kPa}, \geq \mathrm{F} 2 ; 6.8 \mathrm{kPa}, \geq \mathrm{F} 3 ; 8.3 \mathrm{kPa}$ and F4; $14.1 \mathrm{kPa}$.

Blood biomarkers were not as accurate in distinguishing severity of disease. ELF performed better with increasing stages of fibrosis. Area under the curve for cirrhosis was 0.77. CK18-M30 levels could distinguish significant fibrosis $(\geq F 2)(p=0.009)$ with an area under the ROC curve of 0.77 , severe fibrosis ( $\geq$ F3) with an AUROC of 0.69 and cirrhosis (F4) with an AUROC of 0.69. Within the different diagnostic groups, again TE performed better than serum biomarkers with AUROC of 0.75 for $\geq F 2$ and 0.81 for $\geq F 3$ in NAFLD; 0.85 for $\geq$ F2, 0.94 for $\geq$ F3 and 1.0 for F4 in AILD; and in children post-transplant; 0.9 for $\geq \mathrm{F} 2$ and 0.83 for $\geq \mathrm{F} 3$.

Conclusion To our knowledge, this is the largest paediatric study reported to date comparing $\mathrm{TE}$ and serum biomarkers for the non-invasive evaluation of fibrosis. TEwas a reliable tool in distinguishing different stages of liver fibrosis in paediatric patients. Blood biomarkers may be of use in combination with TE especially in the stratification of more severe disease. Routine use of these techniques may serve as a useful adjunct to liver biopsy for diagnostic purposes and provide a reliable method of non-invasively monitoring liver disease progression in children.

\section{P17 TIPS OUTCOMES FOR BUDD-CHIARI: A SINGLE TERTIARY CENTRE EXPERIENCE}

doi:10.1136/gutjnl-2011-300857a.17

\begin{abstract}
${ }^{1} \mathrm{~J}$ Macnaughtan, ${ }^{1} \mathrm{~B}$ Hogan, ${ }^{1} \mathrm{G}$ Tritto, ${ }^{2} \mathrm{M}$ Mullan, ${ }^{2} \mathrm{D} \mathrm{Yu},{ }^{1} \mathrm{D}$ Thorburn, ${ }^{1} \mathrm{~J}$ O'Beirne, ${ }^{1} \mathrm{R}$ Jalan, ${ }^{1} \mathrm{D}$ Patch, ${ }^{1} \mathrm{~A}$ Burroughs. 'Department of Hepatology, Royal Free Hospital; ${ }^{2}$ Department of Radiology, Royal Free Hospital
\end{abstract}

Introduction TIPS insertion is an important intervention in the management of Budd-Chiari syndrome. We describe the experience of a single tertiary referral centre.

Aim The aims of this study were to describe the series of patients undergoing TIPS insertion for Budd-Chiari syndrome at the Royal Free Hospital, survival post-TIPS insertion and re-intervention rates. Method A retrospective analysis of the Royal Free Hospital radiology database was conducted to identify all patients who underwent all
TIPS procedures between January 1991 and January 2011. Patient records were used to subsequently identify those patients in whom Budd-Chiari was the principal indication for TIPS insertion and to characterise this patient cohort.

Results 1073 TIPS-related procedures were conducted at the Royal Free Hospital between January 1991 and January 2011. Of these, 51 patients underwent TIPS insertion for Budd-Chiari syndrome between January 1991 and January 2011 of which 61\% (31/51) were female and $39 \%(20 / 51)$ were male. The mean age at the time of TIPS insertion was 40 years ( \pm 1.96 ). All patients were anticoagulated post procedure. 1-year transplant-free survival post-TIPS insertion was $93 \%$. TIPS insertion could not be achieved in three patients. TIPS stenosis/occlusion was more common than in other TIPS indications. The mean number of TIPS-related interventions was $2.5(1-10)$. Local thrombolysis with tissue plasminogen activator was required in three cases and patency/intervention rates were significantly improved with the addition of asprin to standard warfarin anticoagulation. No patients proceeded to liver transplantation. Regenerative nodules post TIPS were common on surveillance cross sectional imaging.

Conclusion Excellent 1-year transplant-free survival can be obtained in Budd-Chiari syndrome with TIPS placement. Patients are complex and may require multiple interventions and therefore probably best managed in experienced centres.

\section{P18 LIVER FIBROSIS ASSESSED BY TRANSIENT ELASTOGRAPHY PREDICTS RESPONSE IN CHRONIC HEPATITIS C INFECTED PATIENTS TREATED WITH PEG-INTERFERON AND RIBAVIRIN}

doi:10.1136/gutjnl-2011-300857a.18

A N S Nkhoma, D A Sheridan, D A Price, M L Schmid, C Miller, K Baxter M F Bassendine, S McPherson. Freeman Hospital, Joint Viral Hepatitis Clinic

Introduction Chronic hepatitis $\mathrm{C}$ virus infection (HCV) is a common cause of cirrhosis and end stage liver disease. Treatment with Pegylated interferon (PEG-IFN) and ribavirin (RBV) results in sustained virological response (SVR) in approximately $60 \%$ of infected individuals. Increasing stage of fibrosis is known to be a key factor associated with non-response to treatment with PEG-IFN + RBV. Traditionally, fibrosis stage has been determined by liver biopsy, but this is invasive. Newer non-invasive methods of assessing fibrosis such as transient elastography (TE) are now available.

Aim To assess baseline liver stiffness measurement (LSM) assessed by $\mathrm{TE}$ as a predictor of SVR in HCV-infected subjects treated with PEG-IFN+RBV.

Method Retrospective review of outcomes of treatment in naïve patients treated with PEG-IFN+RBV for HCV between 7 January and 9 June. Post-transplant and co-infected patients were excluded. Patients who had valid LSM within 6 months of treatment were included in the TE analysis.

Results 168 patients (mean age 39 $\pm 10,70 \%$ male, 14\% cirrhotic, $53 \%$ high viral load) received PEG-IFN + RBV for HCV in the study period. The overall SVR rate was 59\% (50\% for genotype [G] $1 / 4$ and $70 \%$ for $\mathrm{G} 2 / 3, \mathrm{p}<0.001)$. The SVR rate was only $28 \%$ in cirrhotics (10\% for G1/4 and $43 \%$ for G2/3). 87 patients had a pre-treatment TE (median LSM 6.6 KPa [3.3-73]).

LSM was significantly associated with treatment response to PEGIFN $(p=0.01)$, with the effect more pronounced in HCV G2/3 infection $(p=0.001)$. The optimum cut-off to predict non-response to treatment was $11 \mathrm{KPa} .30$ patients (16\%) stopped treatment due to side-effects or non-compliance, including 1 death from pneumonitis. Conclusion (1) LSM determined by transient elastography may be used as a non-invasive tool to predict treatment response in subjects infected with HCV. 